\title{
A mortalidade hospitalar por causas externas no Brasil e em município do interior de
}

\section{São Paulo}

\author{
Hospital mortality due to external causes in Brazil and in a municipality in the interior of São \\ Paulo
}

Mortalidad hospitalaria por causas externas en Brasil y en un municipio del interior de São Paulo

Recebido: 09/03/2021 | Revisado: 16/03/2021 | Aceito: 17/03/2021 | Publicado: 23/03/2021

\author{
Raquel Cristina Bortolozzo \\ ORCID: https://orcid.org/0000-0002-3104-7798 \\ Fundação Educacional do Município de Assis, Brasil \\ E-mail: raquy_bortolozzo@hotmail.com \\ Júlia Andrade Pires Almeida \\ ORCID: https://orcid.org/0000-0002-1660-3792 \\ Fundação Educacional do Município de Assis, Brasil \\ E-mail: juulia.piires@hotmail.com \\ Ricardo Estefani \\ ORCID: https://orcid.org/0000-0002-0584-673X \\ Fundação Educacional do Município de Assis, Brasil \\ E-mail: estefaniplastica@gmail.com \\ Arlete Aparecida Marçal \\ ORCID: https://orcid.org/0000-0001-8290-9135 \\ Fundação Educacional do Município de Assis, Brasil \\ E-mail: aapmarcal@yahoo.com.br \\ Shirlene Pavelqueires \\ ORCID: https://orcid.org/0000-0002-8740-351X \\ Fundação Educacional do Município de Assis, Brasil \\ E-mail: shirpavelqueires@gmail.com \\ Daniel Augusto da Silva \\ ORCID: https://orcid.org/0000-0002-2716-6700 \\ Fundação Educacional do Município de Assis, Brasil \\ E-mail: daniel.augusto@unifesp.br \\ Caroline Lourenço de Almeida \\ ORCID: https://orcid.org/0000-0002-6043-9301 \\ Fundação Educacional do Município de Assis, Brasil \\ E-mail: caroline_lat@hotmail.com
}

\begin{abstract}
Resumo
As causas externas resultam muitas vezes em internações e óbitos, por esse motivo este trabalho visa o rastreamento das principais e quais são as idades e o sexo mais afetados por elas. Os dados epidemiológicos irão ajudar os profissionais a se prepararem para o melhor atendimentos desses pacientes. De acordo com os números colhidos no DATASUS, a maior causa de internação e óbitos no Brasil e em Assis é a queda, predominando a faixa etária dos idosos. Outra causa que chama muita a atenção é a que engloba os acidentes de transporte, principalmente, os de motocicleta envolvendo o sexo masculino. Espera-se que este estudo possa ajudar na conscientização e na coleta adequada de dados epidemiológicos a respeito do Brasil, suas regiões e Assis.
\end{abstract}

Palavras-chave: Acidentes; Causas externas; Epidemiologia.

\begin{abstract}
External causes often result in hospitalizations and deaths. For this reason, this work aims to track the main causes ande the ages ande sex most affected by them. Epidemiological data will help professionals to prepare for the best care for these patients. According to the figures collected in DATASUS, the major cause of hospitalization and deathes in Brazil and in Assis are the falls, with a predominance of the elderly age group. Another cause that draws a lot of attention is that which includes transport acidentes, especially motorcycle acidentes involving the male sex. It is hoped that this study can help to raise awareness and adequate collection of epidemiological data about Brazil, its regions and Assis. Keywords: Acidentes; External causes; Epidemiology.
\end{abstract}

\section{Resumen}

Las causas externas a menudo resultan en hospitalizaciones y muertes, por lo que este trabajo tiene como objetivo rastrear las principales causas y cuáles son las edades y el sexo más afectados por ellas. Los datos epidemiológicos 
ayudarán a los profesionales a prepararse para la mejor atención para estos pacientes. Según las cifras recogidas en DATASUS, la principal causa de hospitalización y defunciones en Brasil y en Asís es la caída, con predominio del grupo de edad de los ancianos. Otra causa que llama mucho la atención es la que engloba los accidentes de transporte, principalmente los accidentes de motocicleta que involucran al sexo masculino. Se espera que este estudio pueda ayudar a crear conciencia y recopilar adecuadamente datos epidemiológicos sobre Brasil, sus regiones y Assis.

Palabras clave: Accidentes; Causas externas; Epidemiología.

\section{Introdução}

As causas externas influenciaram na dinâmica da mortalidade nas últimas décadas, impulsionada pelas transições demográficas, epidemiológicas e tecnológicas. Nota-se uma redução da mortalidade ocasionadas por doenças transmissíveis e uma elevação desses óbitos por causas externas (Araújo, Menezes, Mendonça, Lopes, Tavares, \& Lima, 2014; Jesus et al., 2020).

A Organização Mundial da Saúde instituiu o termo "causas externas" para as mortes por acidentes e violências, e ocupam o segundo lugar no perfil da mortalidade geral e é a primeira causa de óbitos na faixa etária de 5 a 49 anos. Entre 1991 e 2000, cerca de 1.118 .651 pessoas morreram por esses motivos em nosso país. Desse total, 369.068 foram a óbito por homicídios; 62.480, por suicídio e 309.212, por acidentes e violências no trânsito e nos transportes (Brasil, 2005).

No estado de São Paulo a mortalidade por causas externa foi 22.195 vindo de residência e 21.921 em ocorrência segundo o município. Esses óbitos decorreram de acidente de trânsito, homicídio, suicídio e queimadura em residência (Brasil, 2020). As informações disponíveis sobre óbitos e internações são úteis para monitorar a vigilância epidemiológica das causas externas, contudo, vale destacar que grande parte da demanda de atendimento nos serviços de emergência não são contabilizados - as lesões de menor gravidade (Mascarenhas, Silva, Malta, Moura, Gawryszewski, \& Costa, 2009).

Estimativas apontam que para cada homicídio, 20 a 40 casos de violência não fatal são atendidos pelos serviços de saúde (Cecilio, Grabin, Rovida, Queiróz, \& Grabin, 2012).

Percebe-se na literatura uma vasta publicação referente a mortalidade pós causas externas, porém restringem-se a dados estatístico. Esses dados revelam que as causas mais frequentes são por acidentes, sendo eles, trânsito, quedas e afogamento. São relevantes também os índices em casos de homicídios e suicídios, no qual o predomínio é o sexo masculino (71,5/100mil) na idade de 20 a 60 anos (Corassa, Falci, Gontijo, Machado, \& Alves, 2017; Batista, Barreto, \& Merino, 2018; Figueiredo, Almeida, Martins, \& Silva, 2021).

Dados estes não trazem uma causa principal do Brasil, comparando as regiões, nem o sexo e a faixa etária de maior relevância, ocorrendo perguntas simples e importantes para que através desse achado possa ser realizado uma intervenção: Qual principal causa de óbitos? Com que idade e sexo é mais comum a evolução para óbitos? Que tipo de estratégia poderá reduzir esse índice apresentado? Essas e outras questões merecem estudos profundos, sérios e objetivos.

Diante desses questionamentos, o presente estudo tem como objetivo a caracterização dos atendimentos por causas externas no Brasil e fazendo um comparativo com uma cidade do interior de São Paulo, considerando o fator mortalidade.

Espera-se que com essas informações possamos identificar a realidade da região em relação aos índices de mortalidade por causas externas atendidas na Unidade de Pronto Atendimento e o planejamento de ações de prevenção e estratégias de controle de acordo com os índices identificados como agravantes de mortalidade.

\section{Metodologia}

Trata-se de um estudo epidemiológico, com abordagem quantitativa (Pereira, Shitsuka, Parreira, \& Shitsuka, 2018) realizado por meio de dados que constam no sistema de informação do DATASUS. Fizeram parte do estudo todos óbitos por causas externas, de todas faixas etárias e sexo, no período de novembro de 2019 a abril de 2020 no Brasil e fazendo um comparativo com a cidade de Assis/SP. 
Os critérios de exclusão adotados foram os dados ignorados e/ou inconsistentes na base de dados - DATASUS. As causas externas classificadas foram as seguintes categorias: acidentes (V01-X59), suicídios/lesões autoprovocadas voluntariamente (X60-X84), homicídios/agressões (X85-Y09), indeterminados (eventos/fatos cuja intenção é indeterminada, Y10 a Y34), sendo as demais categorizadas no grupo "outros".

Após a coleta das informações, os dados foram tabulados em planilha eletrônica do Microsoft Office Excel. Empregouse estatística descritiva, com apresentação de frequências absoluta e relativa. No que se refere ao total de óbitos, número e percentual de óbitos, variação percentual (VP) e razão de proporção de óbitos, foram calculados referente ao período de novembro de 2019 a abril de 2020, no intuito de analisar o comportamento da mortalidade, nesse período, entre o Brasil e a cidade de Assis/SP.

A VP refere-se à diferença percentual entre o número absoluto de óbitos em cada capítulo da CID-10 antes (x) e após investigação (y). Para o cálculo da mortalidade proporcional por causas externas foi realizada a divisão do percentual de óbitos do período no Brasil pelo percentual de óbitos do período em Assis/SP.

\section{Resultados e Discussão}

Entre as causas acidentais estavam os acidentes de transporte (V01-V99); as quedas (W00-W19); afogamento e submersão acidentais (W65-W74). A exposição à fumaça/fogo/chamas e envenenamento/ intoxicação por produto químico e/ou substância nociva foram englobadas nos indeterminados em detrimento do desconhecimento da intencionalidade.

Os achados deste estudo revelam que foram notificados, no Brasil e em Assis/SP, 13.272 e 12 óbitos por causas externas, no período de novembro de 2019 a abril de 2020, respectivamente. A região sudeste tem predomino de óbitos (6.051) quando relacionado as demais regiões, seguido da região nordeste (3.280), as faixas etárias mais recorrentes de morte são as mais avançadas, acima de 80 anos, totalizando $21,07 \%$ dos óbitos no país (Tabela 1).

Tabela 1: Distribuição de óbitos por causas externas segundo faixa etária e regiões do Brasil, novembro de 2019 a abril de 2020.

\begin{tabular}{|c|c|c|c|c|c|c|c|}
\hline \multicolumn{8}{|c|}{ Período Analisado: Novembro 2019 - Abril 2020} \\
\hline Faixa Etária 1 & $\begin{array}{l}1 \text { Região } \\
\text { Norte }\end{array}$ & $\begin{array}{l}2 \text { Região } \\
\text { Nordeste }\end{array}$ & $\begin{array}{l}3 \text { Região } \\
\text { Sudeste }\end{array}$ & $\begin{array}{l}4 \text { Região } \\
\text { Sul }\end{array}$ & $\begin{array}{c}5 \text { Região } \\
\text { Centro Oeste }\end{array}$ & Total & $\%$ \\
\hline Menor 1 ano & 8 & 19 & 31 & 13 & 3 & 74 & $0,56 \%$ \\
\hline 01 a 04 anos & 9 & 25 & 35 & 19 & 10 & 98 & $0,74 \%$ \\
\hline 05 a 09 anos & 7 & 17 & 24 & 13 & 3 & 64 & $0,48 \%$ \\
\hline 10 a 14 anos & 3 & 25 & 27 & 10 & 2 & 67 & $0,50 \%$ \\
\hline 15 a 19 anos & 46 & 164 & 198 & 67 & 30 & 505 & $3,81 \%$ \\
\hline 20 a 29 anos & 137 & 487 & 566 & 250 & 100 & 1.540 & $11,60 \%$ \\
\hline 30 a 39 anos & 101 & 420 & 551 & 195 & 104 & 1.371 & $10,33 \%$ \\
\hline 40 a 49 anos & 86 & 380 & 654 & 211 & 109 & 1.440 & $10,85 \%$ \\
\hline 50 a 59 anos & 93 & 410 & 716 & 286 & 114 & 1.619 & $12,20 \%$ \\
\hline 60 a 69 anos & 80 & 380 & 854 & 364 & 109 & 1.787 & $13,46 \%$ \\
\hline 70 a 79 anos & 89 & 399 & 948 & 364 & 110 & 1.910 & $14,39 \%$ \\
\hline 80 anos e mais & 96 & 554 & 1.447 & 538 & 162 & 2.797 & $21,07 \%$ \\
\hline Total Geral & 755 & 3.280 & 6.051 & 2.330 & 856 & 13.272 & $100,00 \%$ \\
\hline
\end{tabular}

Fonte: Autores.

No Brasil, o sexo masculino é predominante nas causas de óbitos, totalizando 68,28\% (Tabela 2). As quedas representam a principal causa de $(18,21 \%)$, seguido de agressões $(8,21 \%)$ no sexo masculino. Já no sexo feminino a causa externa que mais causou óbito também foi a queda $(12,79 \%)$, porém seguido de exposição corrente elétrica $(3,26 \%)$. 
Tabela 2: Distribuição de óbitos por causas externas segundo grupo de causas e sexo no Brasil, novembro de 2019 a abril de 2020 .

\begin{tabular}{|c|c|c|c|c|c|c|}
\hline \multirow[b]{2}{*}{ Grupo de Causas } & \multicolumn{2}{|c|}{ Mascullino } & \multicolumn{2}{|c|}{ Feminino } & \multicolumn{2}{|c|}{ Total } \\
\hline & N & $\%$ & $N$ & $\%$ & $\mathrm{~N}$ & $\%$ \\
\hline V01-V0e Padectre traumatizado sold trancpo rite & 387 & $2,82 \%$ & 164 & $1,16 \%$ & 641 & $4,08 \%$ \\
\hline ViQ-Vie Clolleta traumsizado aold tran cporte & 100 & $0,82 \%$ & 18 & Q.14\% & 127 & $0,96 \%$ \\
\hline V20-Vee Mo to dolleta traumatizado aold tran ip & 844 & $6,26 \%$ & 128 & Q.97\% & 973 & 7,3036 \\
\hline v3o-ve O oup trioldo motortraum aold trancp & 14 & $0,11 \%$ & 1 & Q01\% & 16 & $0,11 \%$ \\
\hline V40.v49 O oup automo vel traum sold trancpo rits & 182 & $1,37 \%$ & 60 & Q.3836 & 282 & $1,76 \%$ \\
\hline V60-V5e O oup ouminhone to traum aold trancports & 3 & $0,02 \%$ & 1 & Q01\% & 4 & $0,08 \%$ \\
\hline ve0-ves o oup velo trancp pecado traum ald tren & 12 & $0,09 \%$ & 2 & Q023 & 14 & $0,11 \%$ \\
\hline V70-V7e o oup dnibuc traumatizado add trancpo it & 4 & $0,03 \%$ & 2 & $0,02 \%$ & 8 & $0,06 \%$ \\
\hline vea-ves Outroc aotd trencporte terre dite & 171 & $1,29 \%$ & 60 & Q.28\% & 221 & $1,8 \% \%$ \\
\hline VeO-Ve4 Aold entec de trancpo rite por aqua & 3 & $0,02 \%$ & 1 & Q01\% & 4 & $0,00 \%$ \\
\hline Ve5-Ve7 Aold entec de trencpo rte asreo/ecpeolal & 2 & $0,02 \%$ & 1 & $0,01 \%$ & 3 & $0,0<\%$ \\
\hline Ves-Ves Outroc ack trancports of n9o ecpe of & 168 & $1,18 \%$ & 69 & Q44\% & 217 & $1,64 \%$ \\
\hline Woo-Wre Qusdac & 2.650 & $10,21 \%$ & 1.697 & $12,78 \%$ & 4.247 & $32,00 \%$ \\
\hline 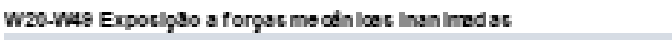 & 280 & $1,86 \%$ & 68 & $0.51 \%$ & 328 & $2,4 \% \%$ \\
\hline W60-WE4 Expoclgeo a forgat me odnloet animadac & 23 & $0,17 \%$ & 6 & Q04\% & 28 & $0,21 \%$ \\
\hline WeE-W74 Afocamento o cubre rebo addentalc & 14 & $0,11 \%$ & 12 & Q00\% & 28 & $0,20 \%$ \\
\hline W76.We4 Outne ricoo c abldentate a recplinglo & $\theta$ & $0,07 \%$ & $B$ & Q05\% & 16 & $0,11 \%$ \\
\hline Was-W6e Expocilgso con rrente eletribo,rad_temp prectso extr & 820 & $8,18 \%$ & 432 & $325 \%$ & 1.252 & $8,43 \%$ \\
\hline xoo-xoe Expoclgas a fumega so fogo e ac ohemse & 88 & $0,68 \%$ & 62 & Q.47\% & 160 & $1,13 \%$ \\
\hline X10-X1e contato fonte de ador a cubstinolac que ntec & 25 & $0,18 \%$ & 13 & Q.10\% & 38 & $0,20 \%$ \\
\hline X20-X28 Contato animalt o plantat venenovor & 31 & $0,23 \%$ & 14 & Q11\% & 45 & $0,34 \%$ \\
\hline X30-X3e Expocigao ac forgact danaturn za & 8 & $0,05 \%$ & 1 & Q01\% & 7 & $0,06 \%$ \\
\hline X40.X49 Envene nementolin toragbo solde ntal expociplo a cubet th & 28 & $0,17 \%$ & 11 & Q08\% & 24 & $0,28 \%$ \\
\hline X60-X67 Exoecto do ecforgot vagent o privapoec & 16 & $0,11 \%$ & 3 & Q02\% & 18 & $0,14 \%$ \\
\hline 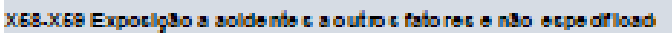 & 808 & $8,08 \%$ & 310 & $224 \%$ & 1.118 & $8,42 \%$ \\
\hline Xe0-X84 Le cBec autop no voced ac volun tarilamente & 116 & $0,87 \%$ & 87 & Q.60\% & 183 & $1,38 \%$ \\
\hline Xs5.YOe Agrectsec & 824 & $6,21 \%$ & 80 & Q.75\% & 823 & $6,66 \%$ \\
\hline Y10.Y34 Eve nto c ou ja linte nglo o ind ete minade & 781 & $6,73 \%$ & 298 & $281 \%$ & 1.147 & $8,84 \%$ \\
\hline Y35.Y3a interve ngbec b galc o operagbec de querra & 2 & $0,02 \%$ & 0 & $0,00 \%$ & 2 & $0,00 \%$ \\
\hline 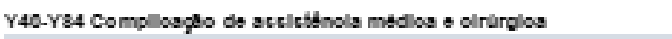 & 397 & $2,89 \%$ & 323 & $243 \%$ & 720 & $6,42 \%$ \\
\hline Ys5.Yse sequelec de osucecexternsc & 284 & $2,22 \%$ & 168 & $1,18 \%$ & 452 & $3,41 \%$ \\
\hline YeQ-Yse Fatorec cuplementarec relaolon alac a outrec osucac & 48 & $0,35 \%$ & 28 & Q.21\% & 74 & $0,60 \%$ \\
\hline 2.T Caucac exte rnac nbo olactiflosdac & 61 & $0,43 \%$ & 47 & $Q 25 \%$ & 108 & $0,81 \%$ \\
\hline Total & 9.062 & $68,28 \%$ & 4.210 & $31,72 \%$ & 13.272 & $100,00 \%$ \\
\hline
\end{tabular}

Assis apresentou um total de 12 óbitos por causas externas durante os meses de novembro de 2019 a abril de 2020 . O sexo masculino foi o único a apresentar registro de óbitos, totalizando $100 \%$. A causa que prevaleceu foi queda (25\%) seguida de acidente com automóvel (16,67\%). (Tabela 3). A faixa etária predominante em Assis foi 50 a 59 anos com 33,3\% do total de óbitos com predomínio de acidentes de ocupante de automóvel, queda e exposição a forças animadas (Tabela 3 ). 
Tabela 3: Distribuição de óbitos por causas externas segundo grupo de causas e sexo em Assis/SP, novembro de 2019 a abril de 2020 .

\begin{tabular}{|c|c|c|c|c|c|c|}
\hline \multirow[b]{2}{*}{ Grupo de Causas } & \multicolumn{2}{|c|}{ Masculino } & \multicolumn{2}{|c|}{ Feminino } & \multicolumn{2}{|c|}{ Total } \\
\hline & $\mathbf{N}$ & $\%$ & $\mathbf{N}$ & $\%$ & $\mathbf{N}$ & $\%$ \\
\hline V01-V09 Pe destre traumatizado acid transporte & 1 & $8,33 \%$ & 0 & $0,00 \%$ & 1 & $8,33 \%$ \\
\hline V10-V19 Ciclista traumatizado acid transporte & 1 & $8,33 \%$ & 0 & $0,00 \%$ & 1 & $8,33 \%$ \\
\hline V40-V49 Ocup automóvel traum acid transporte & 2 & $16,67 \%$ & 0 & $0,00 \%$ & 2 & $16,67 \%$ \\
\hline V80-V89 Outros acid transporte te rrestre & 1 & $8,33 \%$ & 0 & $0,00 \%$ & 1 & $8,33 \%$ \\
\hline Woo-W19 Quedas & 3 & $25,00 \%$ & 0 & $0,00 \%$ & 3 & $25,00 \%$ \\
\hline W50-W64 Exposição a forças me cânicas animadas & 1 & $8,33 \%$ & 0 & $0,00 \%$ & 1 & $8,33 \%$ \\
\hline X85-Y09 Agressões & 2 & $16,67 \%$ & 0 & $0,00 \%$ & 2 & $16,67 \%$ \\
\hline Y40-Y84 Complicação de assistência médica e cirúrgica & 1 & $8,33 \%$ & 0 & $0,00 \%$ & 1 & $8,33 \%$ \\
\hline Total & 12 & $100,00 \%$ & 0 & $0,00 \%$ & 12 & $100,00 \%$ \\
\hline
\end{tabular}

Fonte: Autores.

Comparando os óbitos por causas externas no Brasil e na cidade de Assis/SP, considerando a faixa etária, nota-se um predomínio de óbitos em pessoas acima de 80 anos no Brasil (28,8\%), em Assis a faixa etária mais atingida está entre os 50 a 59 $\operatorname{anos}(33,3 \%)$ (Figura 1). Analise importante é que em segundo lugar a idade que prevalece na cidade de Assis está entre 15-19 anos e 40-49 anos, ambos com 16,7\% dos óbitos no período.

Figura 1: Número de óbitos por causas externa em porcentagem comparativa na cidade de Assis/SP e no Brasil, novembro de 2019 a abril de 2020 .

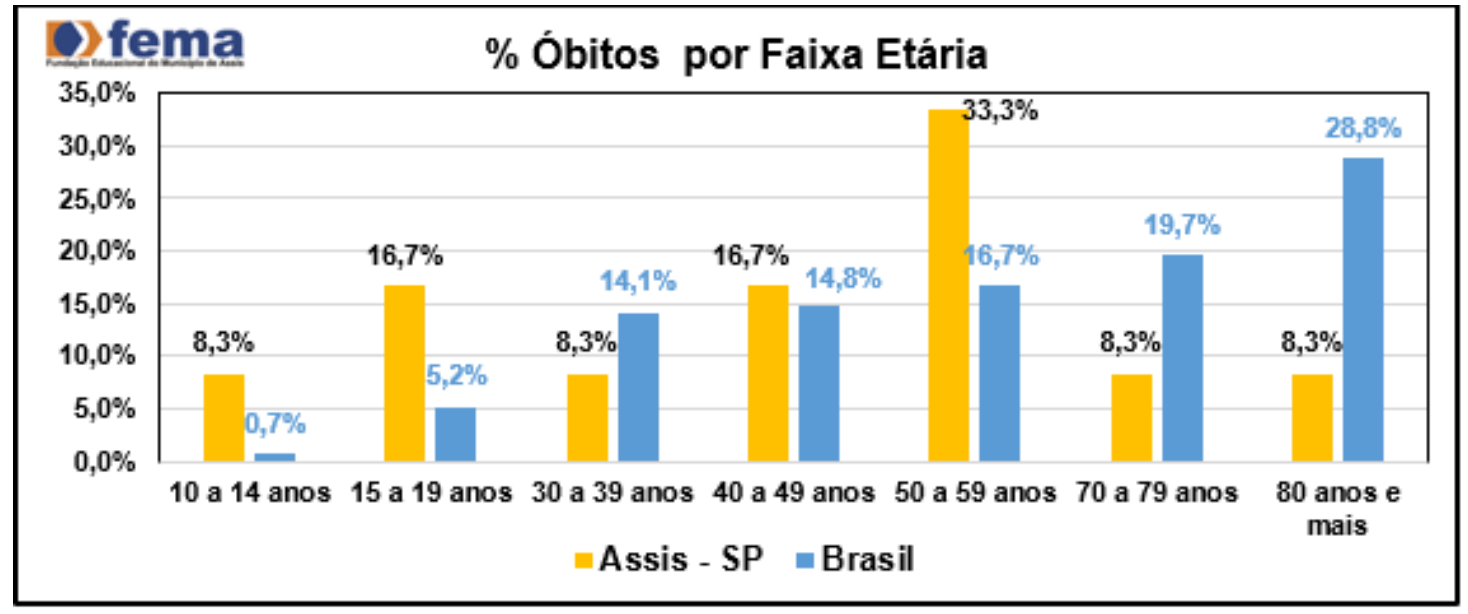

Fonte: Autores.

Entende-se que a questão de a região sudeste ser a mais populosa do país acarreta maior número de internações e mortalidade. Com relação ao sexo destaca-se o masculino por todo Brasil e na cidade de Assis, corroborando com outros locais no mundo como na Australia (51,4\%) (Australian Bureau of Statistics, 2011) e na Polônia (75,2\%) (Pikala, Bryla, Bryla, \& Maniecka-Bryla, 2014). Isto evidencia a vulnerabilidade desse sexo em questão de agravos e maior exposição a comportamentos e atividades de maior risco (Nery, Alves, Rios, Assunção, \& Matos Filho, 2013).

Em relação com a principal causa de óbitos, quedas, e as faixas etárias observa um predomínio de idosos que estão em condições predisponentes para as quedas como, por exemplo, alterações anatômicas, fisiológicas e físicas decorrentes do processo de envelhecimento associado a doenças crônicas, comorbidades e barreiras arquitetônicas (Gawryszewski, Hidalgo, \& 
Valencich, 2012; Silva, Silva, Ferreira, Castro, \& 2020; Silveira \& Silva, 2020).

Essas questões se relacionam diretamente às transições demográfica e epidemiológica, que observamos atualmente, e levam à maior expectativa de vida e um maior contingente populacional entre as pessoas com mais de sessenta anos (Batista, Barreto, \& Merino, 2018; Silva, Silva Neto, \& Vidal, 2020).

Os índices de mortalidade por causas acidentais e violentas são influenciados pelos determinantes socioculturais e têm sido associados com o modelo escolhido para o sistema de transporte prioritário nas estradas e no uso de carros privados sem oferecer infraestrutura rodoviária adequada (Santos, Maia, Diniz, Santos, \& Pimenta, 2013; Moreira, Santos, Oliveira Neto, \& Silva Junior, 2019).

\section{Considerações Finais}

Este estudo concluiu que as vítimas de causas externas no Brasil e na cidade de Assis/SP foi, no período de estudo, homens em todas as causas pesquisadas. As causas acidentais prevaleceram em relação às violências e, entre as causas específicas, destacaram-se as quedas, nos dois locais pesquisados, e as agressões e acidentes de transporte em Assis/SP.

Os resultados apontaram um dado importante em relação a faixa etária dos óbitos por causas externas na cidade de Assis/SP, nota-se um predomínio em idades jovens, fato que pode estar relacionado por acidentes e violências na cidade. Esperase que esta pesquisa possa permitir análises para subsidiar melhorias na qualidade da notificação destes casos, bem como incitar medidas preventivas com a população, para redução dos óbitos por causas externas no país e na cidade em questão.

\section{Referências}

Araújo, A. M., Menezes, R. M. P., Mendonça, A. E. O., Lopes, M. S., Tavares, A. M., \& Lima, H. C. F. (2014). Mortality profile from falls in the elderly. Revista de Pesquisa: Cuidado Fundamental, 6(3), 863-875.

Australian Bureau of Statistics. (2011). Causes of death, Australia. Australian Bureau of Statistics.

Batista, J., Barreto, M. S., \& Merino, M. F. G. L. (2018). Perfil Epidemiológico da Mortalidade por Causas Externas Entre Beneficiários de Planos de Saúde no Brasil. Revista de Enfermagem do Centro-Oeste Mineiro, 8.

Brasil. Ministério da Saúde. (2020). Sistema de Informações Hospitalares do Sus (SIH/SUS/DATASUS). http://tabnet.datasus.gov.br/cgi/deftohtm.exe?sih/cnv/fiuf.def.

Brasil. Ministério da Saúde. (2005). Impacto da violência na saúde dos brasileiros. Ministério da Saúde.

Cecilio, L. P. P., Grabin, C. A. S., Rovida, T. A. S., Queiróz, A. P. D. G., \& Grabin, A. J. I. (2012). Violência interpessoal: estudo descritivo dos casos não fatais atendidos em uma unidade de urgência e emergência de sete municípios do Estado de São Paulo, Brasil, 2008 a 2010. Epidemiologia e Serviço de Saúde, 21(2), 293-304.

Corassa, R. B., Falci, D. M., Gontijo, C. F., Machado, G. V. C., \& Alves, P. A. B. (2017). Evolução da mortalidade por causas externas em Diamantina (MG), 2001 a 2012. Caderno de saúde coletiva, 25(3), 302-314.

Figueiredo, E. A., Almeida, C. L., Martins, E. A. P., \& Silva, D. A. (2021). Acidente motociclístico: caracterização dos atendimentos pré-hospitalares no interior de São Paulo. Research, Society and Development, 10(2), e37410212575. https://doi.org/10.33448/rsd-v10i2.12575

Gawryszewski, V. P., Hidalgo, N. T., \& Valencich, D. M. O. (2012). A queda nas taxas de homicídios no estado de São Paulo e apresentação dos dados de mortalidade por causas externas em 2004. Boletim Epidemiológico Paulista, 2(21).

Jesus, P. N. L. de, Macedo, G. B., Osternes, F. N. D., Pereira, K. L. A., Macedo, D. B., Ferreira, M. das G. S. dos S., Maia, J. B. D., Oliveira, M. M. L. de D., \& Macedo, J. B. (2020). Sintomas depressivos em familiares de jovens que tiveram óbito devido a causas externas. Research, Society and Development, 9(10), e8459109134. https://doi.org/10.33448/rsd-v9i10.9134

Ministério da Saúde. (2016). Estatísticas de mortalidade em beneficiários de saúde suplementar: óbitos por residência segundo causas externas 2005 -2009. Brasília, Ministério da Saúde.

Moreira, L. de A., Santos, S. F. dos, Oliveira Neto, R. de, \& Silva Junior, L. A. (2019). Revisão bibliográfica sobre o modal de transporte rodoviário no Brasil. Research, Society and Development, 8(3), e2283728. https://doi.org/10.33448/rsd-v8i3.728

Nery, A. A., Alves, M. S., Rios, M. A., Assunção, P. N., \& Matos Filho, S. A. (2013). Perfil epidemiológico da morbimortalidade por causas externas em um hospital geral. Revista de Enfermagem. UFPE on-line, 7(2), 562-571.

Pereira, A. S., Shitsuka, D. M., Parreira, F. J., \& Shitsuka, R. (2018). Metodologia da pesquisa científica. UFSM. 
Research, Society and Development, v. 10, n. 3, e49710313707, 2021

(CC BY 4.0) | ISSN 2525-3409 | DOI: http://dx.doi.org/10.33448/rsd-v10i3.13707

Pikala, M, Bryla, M, Bryla, P, \& Maniecka-Bryla, I. (2014). Years of life lost due to external causes of death in the Lodz Province, Poland. PLos ONE, 9(5), e96830.

Santos, V. R., Maia, C. S., Diniz, C. G., Santos, B. F., \& Pimenta, A. M. (2013). Morbimortalidade de usuários de um plano privado de saúde de Belo Horizonte, Minas Gerais. Revista de Enfermagem do Centro-Oeste Mineiro, 3(3), 788-796.

Silva, J. P. F., Silva Neto, F. S., \& Vidal, G. P. (2020). Recursos fisioterapêuticos empregados na prevenção de quedas na população idosa. Research, Society and Development, 9(8), e192985567. https://doi.org/10.33448/rsd-v9i8.5567

Silva, L. D., Silva, N. C., Ferreira, E. S., Castro, L. C., \& Alencar, M. S. S. (2020). Fatores de risco que potencializam fragilidades em idosos institucionalizados. Research, Society and Development, 9(5), e57953189. https://doi.org/10.33448/rsd-v9i5.3189

Silveira, A. G., \& Silva, D. A. (2020). Sobrecarga dos familiares no cuidado ao portador de demência senil: uma revisão integ rativa. Research, Society and Development, 9(6), e179963671. https://doi.org/10.33448/rsd-v9i6.3671 